\title{
Are Schools Really Like This?
}

Factors Affecting Teacher Attitude toward School Improvement 


\section{INNOVATIONS IN SCIENCE EDUCATION AND TECHNOLOGY}

\section{Series Editor:}

Karen C. Cohen, Harvard University, Cambridge, Massachusetts

Are Schools Really Like This?: Factors Affecting Teacher Attitude toward School Improvement

J. Gary Lilyquist

The Hidden Curriculum-Faculty-Made Tests in Science Part 1: Lower-Division Courses

Part 2: Upper-Division Courses

Sheila Tobias and Jacqueline Raphael

Internet Links for Science Education: Student-Scientist

Partnerships

Edited by Karen C. Cohen

Web-Teaching: A Guide to Designing Interactive Teaching for the World Wide Web

David W. Brooks 


\title{
Are Schools Really Like This?
}

Factors Affecting Teacher Attitude toward School Improvement

\author{
J. Gary Lilyquist \\ Independent Quality Consultant in Education \\ Milltown, Wisconsin
}

Springer Science+Business Media, LLC 


\section{Library of Congress Cataloging-in-Publication Data}

Lilyquist, J. Gary

Are schools really like this? : factors affecting teacher attitude toward school improvement / J. Gary Lilyquist.

p. cm. - (Innovations in science education and technology)

Includes bibliographical references and index.

1. School improvement programs-United States-Case studies. 2. Teachers-United States-Attitudes - Case studies. 3. School environment-United States-Case studies.

4. Teacher participation in administration-United States-Case studies. I. Title.

II. Series

LB2822.82.L55 1998

97-40389

$371.1^{\prime} 06-\mathrm{dc} 21$

\section{ISBN 978-0-306-45735-7 ISBN 978-1-4757-9282-9 (eBook)}

DOI 10.1007/978-1-4757-9282-9

(C) 1998 Springer Science+Business Media New York

Originally published by Plenum Press, New York in 1998.

\section{1}

\section{All rights reserved}

No part of this book may be reproduced, stored in a retrieval system, or transmitted in any form or by any means, electronic, mechanical, photocopying, microfilming, recording, or otherwise, without written permission from the Publisher 
This book is dedicated to the improvement of learning opportunities for all children 


\section{Foreword}

This is a hopeful book with a message that should cheer those who have devoted their lives to public schools. It emphatically states that the system should be saved, although it affirms that change is needed. It is about how to reconcile differing visions of school through an understanding of what is involved in the working of schools and of the nature of change.

The book's several strengths make it useful both to readers who are school people as well as to those who are not. It really does describe what schools are like through a deep and detailed discussion of three case studies. It actually brings systems thinking and a review of the research into school work in an attempt to analyze how to make needed and lasting changes.

Its personal, interactive style engages the reader in a conversation about how to change schools to improve student learning. It ventures into the realm of teacher status while clearly warning us not to blame the people. It stresses the complexity of the institution and its interdependence with its client groups.

It is clearly a book by someone who respects and enjoys working with schools and the people who are connected with them.

Isa Kaftal Zimmerman, Superintendent Acton Public Schools

Acton-Boxborough Regional School District Acton, Massachusetts 


\section{Preface}

Systems thinking, already an established movement in many businesses and industries, has only recently been introduced into the educational system. This book is intended to provide a framework for educators, teachers-in-training, educational administrators, parents, community members, government leadership, and policy makers. To successfully carry out school improvements, the framework combines the concepts of systems thinking, mental models, effective school research, and Quality Management Theory. Using analysis of six organizational dimensions (internal and external cultures, leadership, strategy, structure, and results), three case studies show the attempts of school systems to balance the culture's visions of a good school. The book also comments on how these individuals and groups could apply more systems thinking.

The examples of three school cultures that are likely to be familiar to the reader offer a different way to approach school improvement. First, the book offers in a systemic format a rationale as to why people react (positively and negatively) to school improvement. Second, it compares three case studies that support suggestions of why schools are not improving. Third, it suggests a new approach, a Balance Alignment Model, for systemic school improvement. The concluding chapters discuss three key boundaries that cannot be ignored if we want to carry out successful projects to increase student learning. Otherwise these boundaries may contradict popular ideas about how to improve our schools. 


\section{Acknowledgments}

Three people have had great influence on me, shaping my thinking on management theory and leading to completion of this book. First, Professor Jacob O. Stampen, Department of Educational Administration, University of WisconsinMadison, offered continued guidance and a nonjudgmental style of teaching, creating a learning condition in which I was free to explore and challenge my mental model on management theory. Second, Professor B. Dean Bowles challenged my understanding of Quality Management Theory and its compatibility with education's political system. Third, Professor Mark P. Finster, Grainger School of Business, in the Center for Quality and Productivity Improvement, introduced me to the Masters of Quality Improvement Theory.

I value the opportunity to have worked with Dr. Betty B. Hoskins, the Developmental Editor. She was very helpful and supportive of changes in the text.

Without the support of JoAnne, I would not have had the opportunity to write this book. 


\section{Contents}

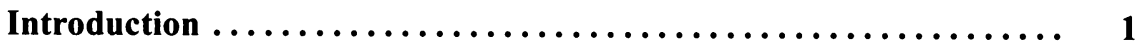

Research Method $\ldots \ldots \ldots \ldots \ldots \ldots \ldots \ldots \ldots \ldots \ldots \ldots \ldots, \ldots \ldots \ldots$

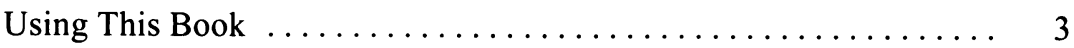

1. Vision of a Good School $\ldots \ldots \ldots \ldots \ldots \ldots \ldots \ldots \ldots \ldots \ldots \ldots, 7$

The Teachers' Paradox ............................ 8

The Paradox and the Community .................... 9

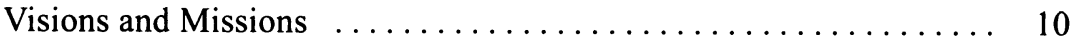

2. Systems Thinking $\ldots \ldots \ldots \ldots \ldots \ldots \ldots \ldots \ldots \ldots \ldots, 13$

What Can Be Done? .......................... 15

3. Building a Systemic Model $\ldots \ldots \ldots \ldots \ldots \ldots \ldots \ldots \ldots \ldots \ldots, 17$

"Special Cause" and "Common Cause" Perspectives $\ldots \ldots \ldots \ldots .18$

"Common Cause" Problems ........................... 18

The Balance Model (Lilyquist, 1995) .................. 19

The Concept of Balance ......................... 23

Summary $\ldots \ldots \ldots \ldots \ldots \ldots \ldots \ldots \ldots \ldots \ldots \ldots \ldots \ldots \ldots \ldots, 24$

4. Systemic Alignment and Balance $\ldots \ldots \ldots \ldots \ldots \ldots \ldots \ldots \ldots, 25$

Leadership (Formal and Informal) ................... 25

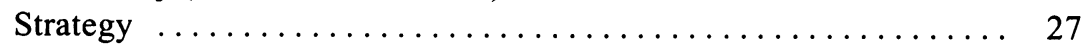

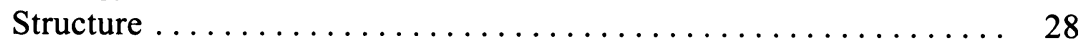

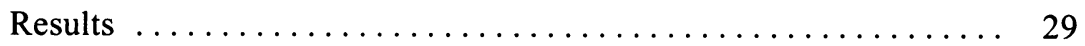

Alignment and Balance $\ldots \ldots \ldots \ldots \ldots \ldots \ldots \ldots \ldots \ldots, \quad 30$ 
5. Stakeholders I: Community, Community Members ......... 33

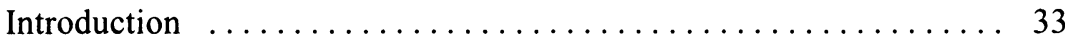

Community as External Environment (Culture) .............. 33

Case Study One - Dryden ........................ 34

Stability .............................. 34

Pressure ................................ 34

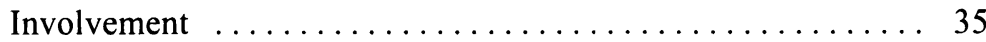

Comment ............................... 36

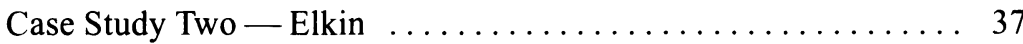

Stability ............................... 37

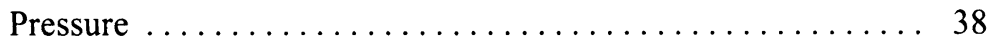

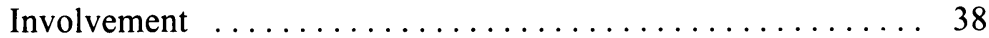

Comment ................................. 39

Case Study Three - Skyland $\ldots \ldots \ldots \ldots \ldots \ldots \ldots \ldots \ldots . \ldots \ldots$

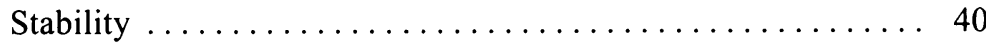

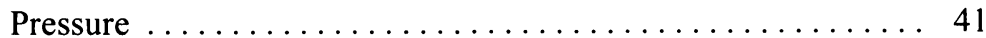

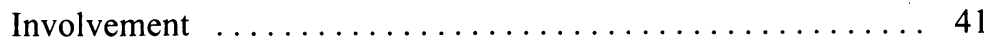

Comment ............................. 43

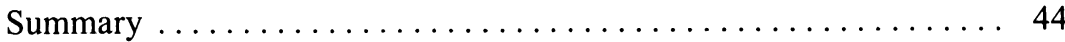

Comparison of the Communities' Political Power Structure ........ 44

Community Visions $\ldots \ldots \ldots \ldots \ldots \ldots \ldots \ldots \ldots \ldots \ldots \ldots$

\section{Stakeholders II: Parents, Students, and State and National}

Government ............................... 47

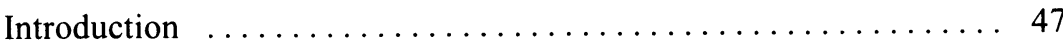

Parental Control and Involvement $\ldots \ldots \ldots \ldots \ldots \ldots \ldots \ldots 4$

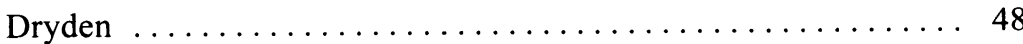

Elkin .................................... 49

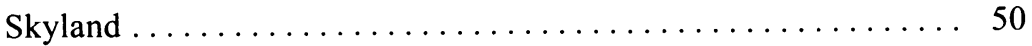

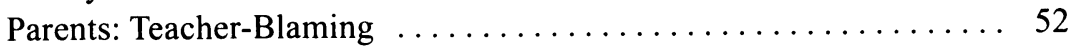

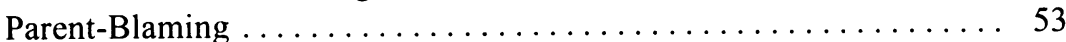

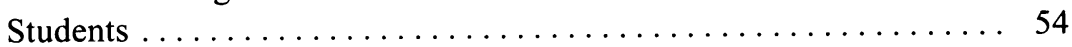

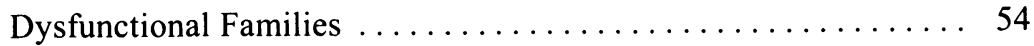

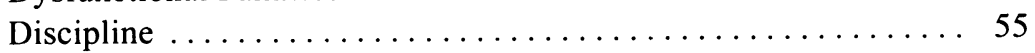

Special Needs Students ........................ 57

State and National Government $\ldots \ldots \ldots \ldots \ldots \ldots \ldots \ldots \ldots$

State Government: Funding $\ldots \ldots \ldots \ldots \ldots \ldots \ldots \ldots \ldots \ldots \ldots$

State Government: Mandates ..................... 60

National Government and Media $\ldots \ldots \ldots \ldots \ldots \ldots \ldots \ldots . \ldots 6$

Closing Comments on the External Environment ........... 62 
7. The Closed Culture $\ldots \ldots \ldots \ldots \ldots \ldots \ldots \ldots \ldots \ldots \ldots \ldots, 63$

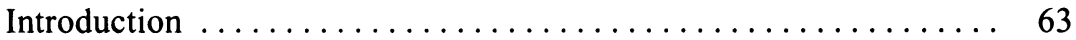

Internal Environment Culture $\ldots \ldots \ldots \ldots \ldots \ldots \ldots \ldots \ldots \ldots$

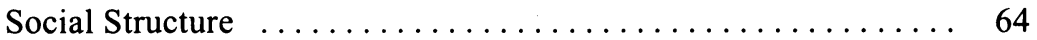

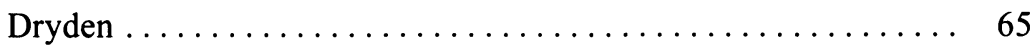

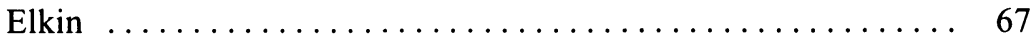

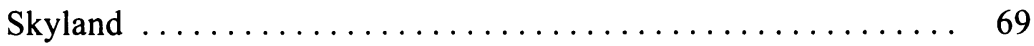

Support System ........................... 71

Dryden ................................... 72

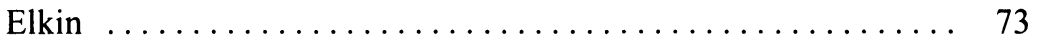

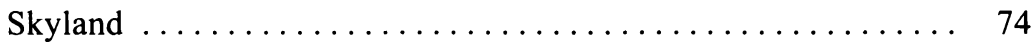

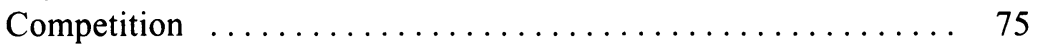

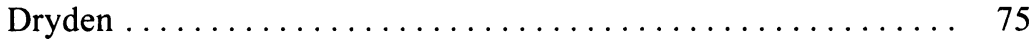

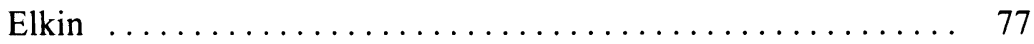

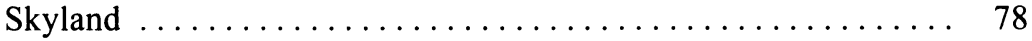

Being Valued in Decision-Making $\ldots \ldots \ldots \ldots \ldots \ldots \ldots \ldots$

Dryden .................................. 79

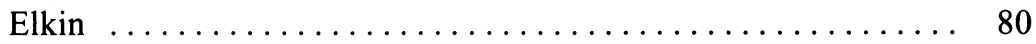

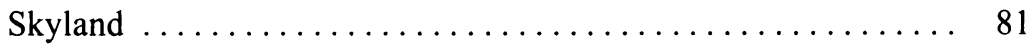

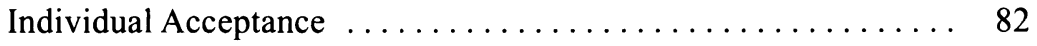

Dryden ................................ 82

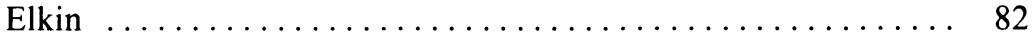

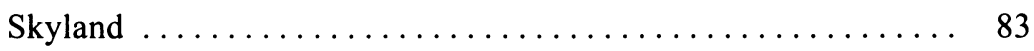

Trust in Administrative Leadership ................... 84

Dryden ................................ 84

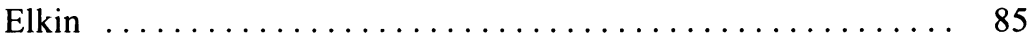

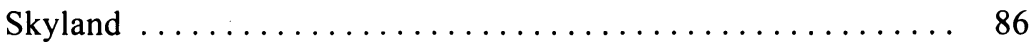

Hardworking Teachers $\ldots \ldots \ldots \ldots \ldots \ldots \ldots \ldots \ldots \ldots \ldots \ldots$

Dryden ................................. 87

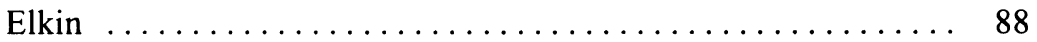

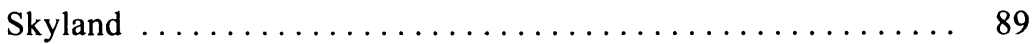

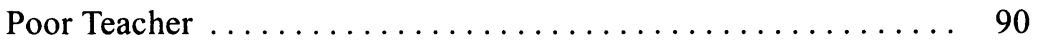

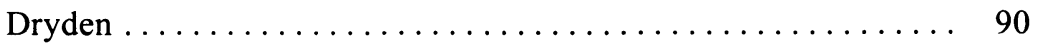

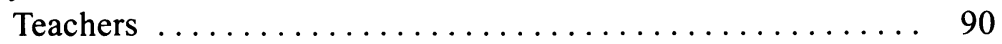

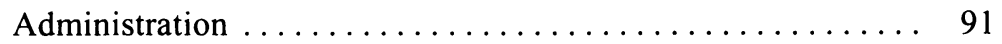

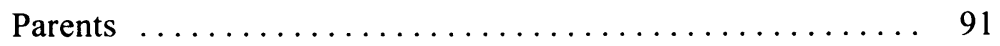

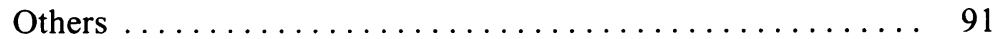

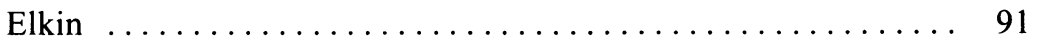

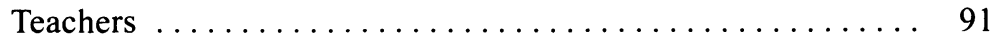

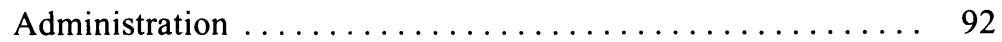

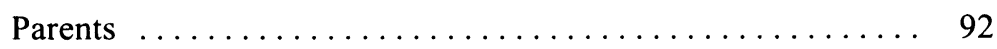

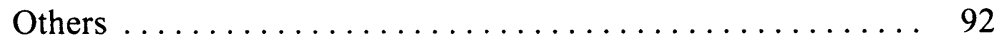




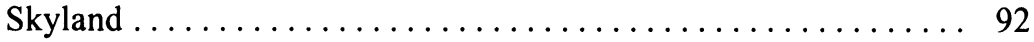

Teachers ............................ 92

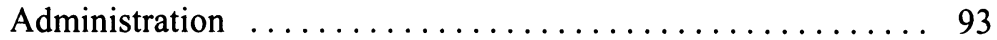

Parents ................................ 93

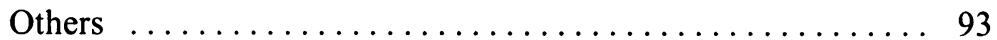

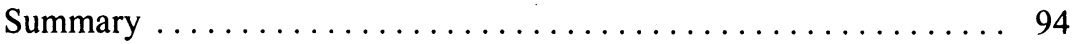

Comment .................................. 94

8. Leadership $\ldots \ldots \ldots \ldots \ldots \ldots \ldots \ldots \ldots \ldots \ldots \ldots \ldots \ldots \ldots, 95$

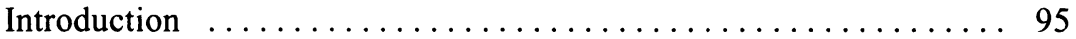

Leadership Dimension $\ldots \ldots \ldots \ldots \ldots \ldots \ldots \ldots \ldots \ldots \ldots$

Top-Down Management $\ldots \ldots \ldots \ldots \ldots \ldots \ldots \ldots \ldots \ldots$

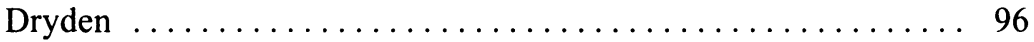

Elkin ................................... 97

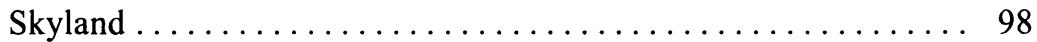

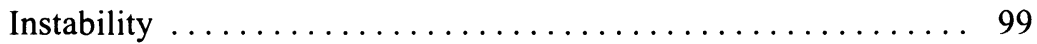

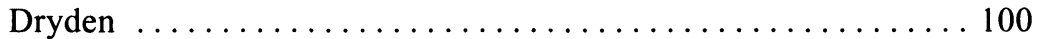

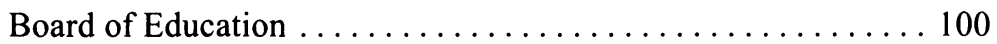

Changing Leadership ........................ 101

Powerlessness ................................. 101

Administration Protecting Themselves ............... 101

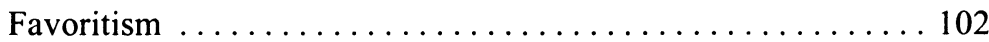

Elkin .................................... 102

Board of Education ....................... 102

Changing Leadership $\ldots \ldots \ldots \ldots \ldots \ldots \ldots \ldots \ldots \ldots$

Powerlessness .............................. 103

Administration Protecting Themselves ............... 104

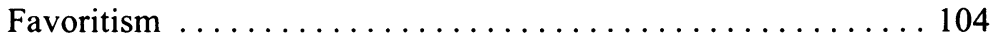

Skyland ..................................... 104

Board of Education ......................... 104

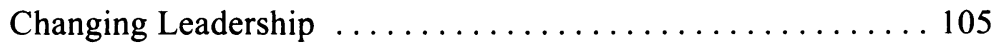

Powerlessness ............................ 105

Administration Protecting Themselves .............. 105

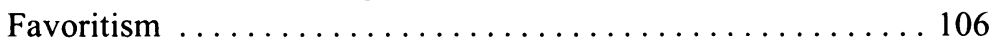

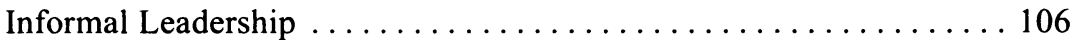

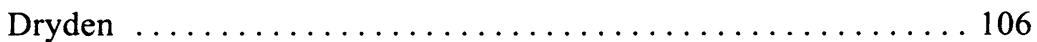

Elkin .................................... 107

Skyland .................................. 108

Summary $/$ Comment ........................... 109 
9. Complete, Incomplete, and Thwarted Planning ........... 111

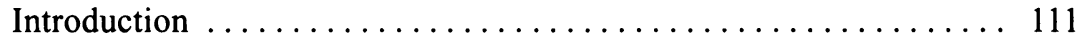

Strategy Dimension $\ldots \ldots \ldots \ldots \ldots \ldots \ldots \ldots \ldots \ldots \ldots \ldots \ldots \ldots \ldots$

Unclear Purpose ........................ 112

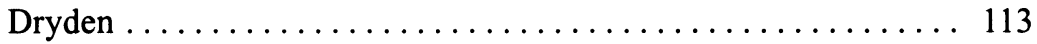

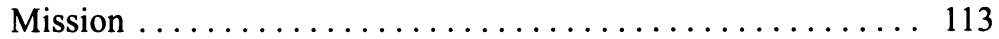

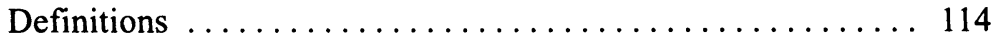

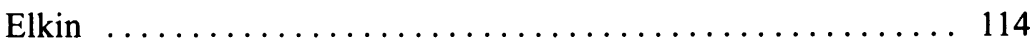

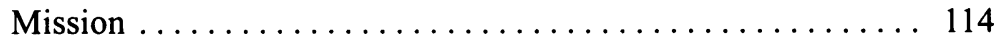

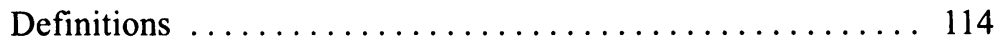

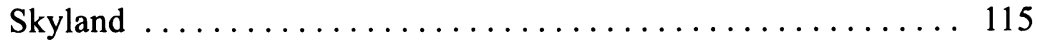

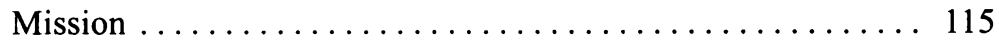

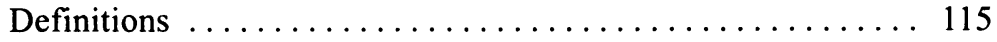

Guidelines ............................ 116

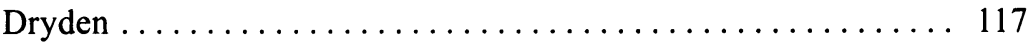

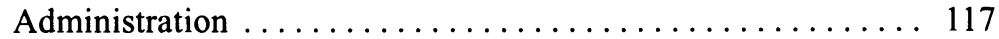

Teachers ............................. 118

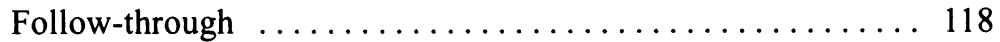

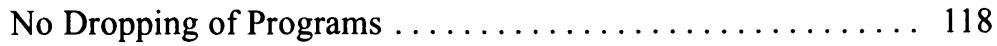

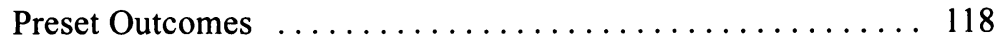

Elkin ............................ 119

Administration ...................... 119

Teachers ......................... 119

Follow-through $\ldots \ldots \ldots \ldots \ldots \ldots \ldots \ldots \ldots \ldots \ldots \ldots$

No Dropping of Programs $\ldots \ldots \ldots \ldots \ldots \ldots \ldots \ldots \ldots \ldots$

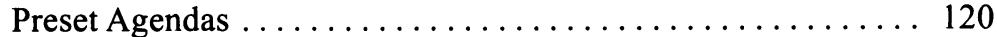

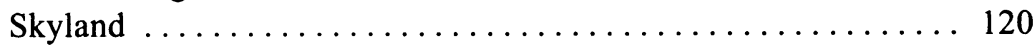

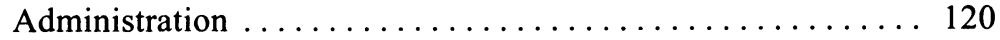

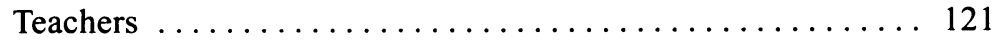

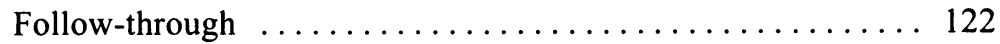

No Dropping of Programs . . . . . . . . . . . . . . 122

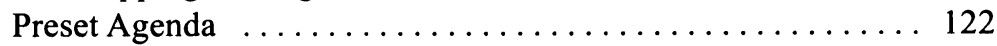

Summary/Comment $\ldots \ldots \ldots \ldots \ldots \ldots \ldots \ldots \ldots \ldots \ldots \ldots \ldots$

10. The Structure $\ldots \ldots \ldots \ldots \ldots \ldots \ldots \ldots \ldots \ldots \ldots \ldots \ldots \ldots$

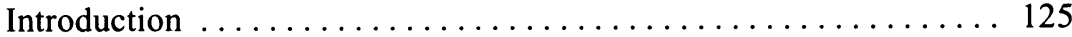

Structure Dimension ........................ 126

Small Groups and Individual Influence $\ldots \ldots \ldots \ldots \ldots \ldots \ldots$

Dryden . . . . . . . . . . . . . . . . . . . . . . . . . . 127

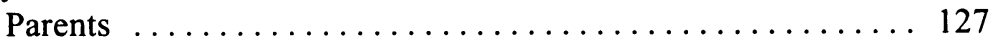

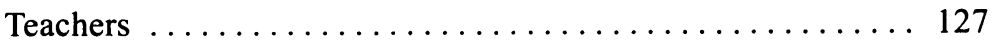


Administration ....................... 127

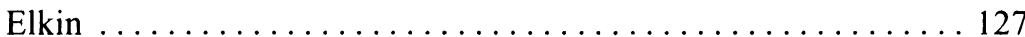

Parents ............................... 127

Teachers .......................... 128

Administration .......................... 129

Skyland ... . . . . . . . . . . . . . . . . . . . . . . . . . . 129

Parents .................................. 129

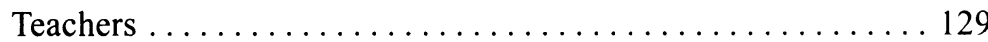

Administration ............................ . 129

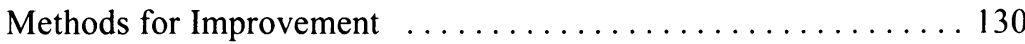

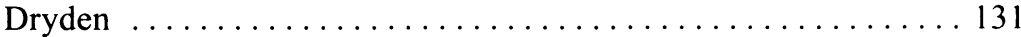

Committees .......................... 131

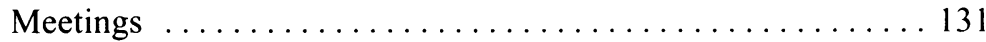

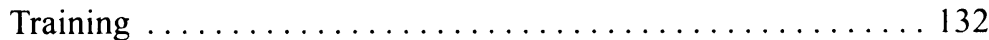

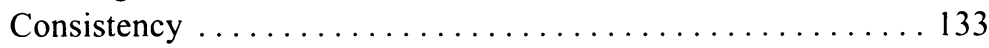

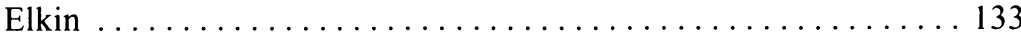

Committees ........................... 133

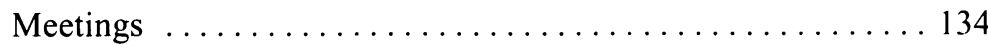

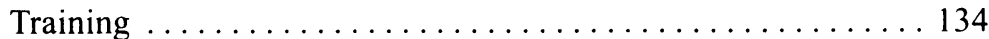

Consistency ............................. 135

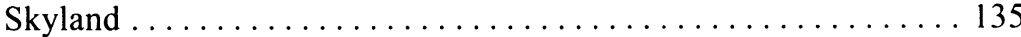

Committees .......................... 135

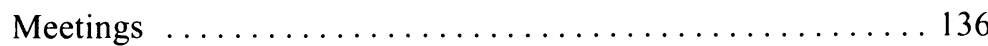

Training ............................ 137

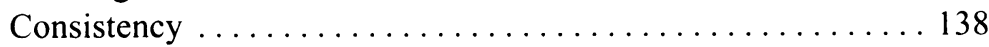

Instructional Coordination $\ldots \ldots \ldots \ldots \ldots \ldots \ldots \ldots \ldots \ldots \ldots$

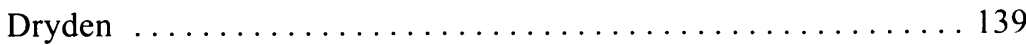

Elkin .............................. 441

Skyland ............................. 142

Administration ........................... 143

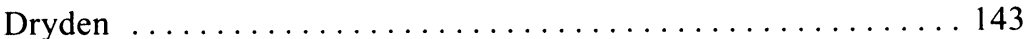

Decision-Makers . . . . . . . . . . . . . . . . . . . . 143

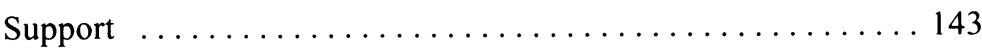

Elkin ................................. . . 144

Decision-Makers ... . . . . . . . . . . . . . . . . . . . . . 144

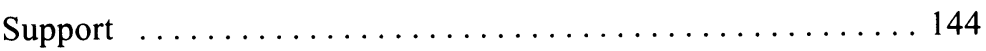

Skyland ............................... 144

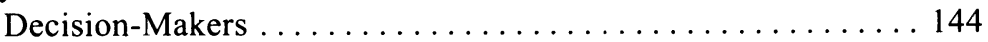

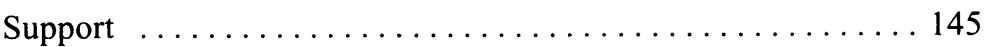

Conflicting Needs: School and Home . . . . . . . . . . . . . 145

Dryden ............................ 146

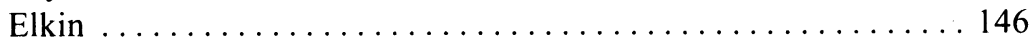

Skyland ............................ 147 
Other School Services $\ldots \ldots \ldots \ldots \ldots \ldots \ldots \ldots \ldots \ldots \ldots . \ldots 148$

Dryden .................................. 148

Elkin ................................... 149

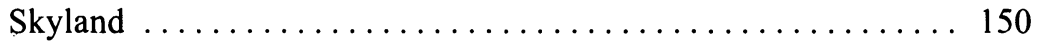

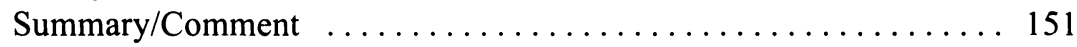

11. Perception or Reality $\ldots \ldots \ldots \ldots \ldots \ldots \ldots \ldots \ldots \ldots \ldots \ldots \ldots \ldots$

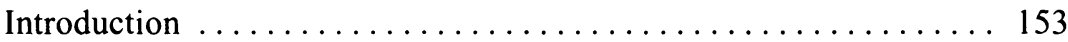

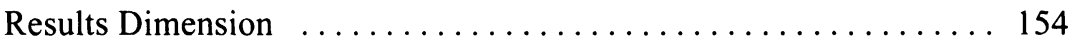

Use of Data ................................. 154

Dryden ................................... 154

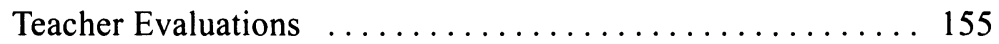

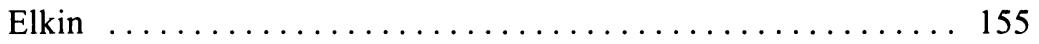

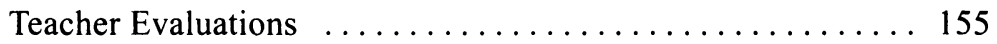

Skyland ..................................... 156

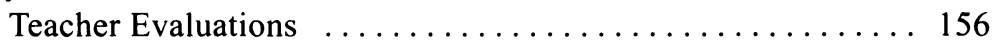

Evaluation of Instruction ....................... 157

Dryden ................................... 157

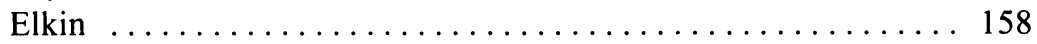

Skyland .................................... 159

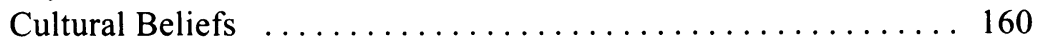

Dryden .................................... 161

Classroom Teachers ......................... 161

Special Education Teachers $\ldots \ldots \ldots \ldots \ldots \ldots \ldots \ldots \ldots . \ldots \ldots 2$

Others ................................ 162

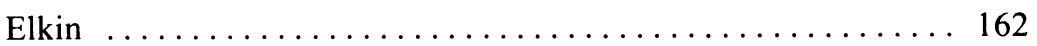

Classroom Teachers ....................... 162

Special Education Teachers $\ldots \ldots \ldots \ldots \ldots \ldots \ldots \ldots \ldots . \ldots \ldots$

Others .................................. 163

Skyland .................................... 164

Classroom Teachers .......................... 164

Special Education Teachers $\ldots \ldots \ldots \ldots \ldots \ldots \ldots \ldots$

Others .................................... 165

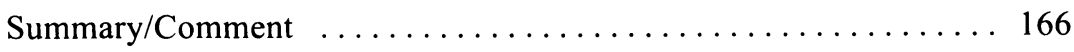

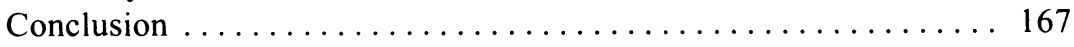

12. Thoughts on School Management $\ldots \ldots \ldots \ldots \ldots \ldots \ldots \ldots \ldots . \ldots$

Introduction . . . . . . . . . . . . . . . . . . . . . . . . 169

Literature on School Improvement $\ldots \ldots \ldots \ldots \ldots \ldots \ldots \ldots \ldots$

Approaches to School Improvement ................ 170 
Effective Schools ......................... 170

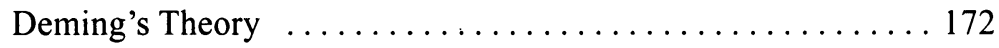

The Balance Alignment Model and Theory $\ldots \ldots \ldots \ldots \ldots \ldots \ldots$

Concluding Comments and Suggestions for Further Research ...... 176

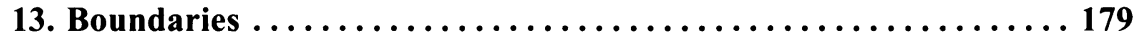

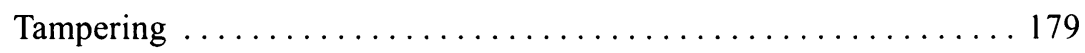

The Challenges $\ldots \ldots \ldots \ldots \ldots \ldots \ldots \ldots \ldots \ldots \ldots \ldots \ldots \ldots$

References $\ldots \ldots \ldots \ldots \ldots \ldots \ldots \ldots \ldots \ldots \ldots \ldots \ldots \ldots \ldots \ldots \ldots \ldots, 183$

Index $\ldots \ldots \ldots \ldots \ldots \ldots \ldots \ldots \ldots \ldots \ldots \ldots \ldots \ldots \ldots \ldots \ldots . \ldots \ldots$ 